\title{
ALMOST EVERYWHERE POSITIVITY OF THE LYAPUNOV EXPONENT FOR THE DOUBLING MAP
}

\author{
DAVID DAMANIK AND ROWAN KILLIP
}

\begin{abstract}
We show that discrete one-dimensional Schrödinger operators on the half-line with ergodic potentials generated by the doubling map on the circle, $V_{\theta}(n)=f\left(2^{n} \theta\right)$, may be realized as the half-line restrictions of a nondeterministic family of whole-line operators. As a consequence, the Lyapunov exponent is almost everywhere positive and the absolutely continuous spectrum is almost surely empty.
\end{abstract}

Consider discrete Schrödinger operators

$$
\left[H_{\theta} \phi\right](n)=\phi(n+1)+\phi(n-1)+\lambda f\left(2^{n} \theta\right) \phi(n)
$$

on $\ell^{2}\left(\mathbb{Z}_{+}\right), \mathbb{Z}_{+}=\{1,2, \ldots\}$, with a Dirichlet boundary condition, $\phi(0)=0$. Here, $\lambda>0$ denotes the coupling constant, $\theta \in \mathbb{T}=\mathbb{R} / \mathbb{Z}$, and $f: \mathbb{T} \rightarrow \mathbb{R}$ is measurable, bounded, and non-constant.

Since the doubling map, $T: \mathbb{T} \rightarrow \mathbb{T}, \theta \mapsto 2 \theta \bmod 1$, is ergodic with respect to Lebesgue measure on $\mathbb{T}$, the Lyapunov exponent exists for every energy $E$, that is, there is a function $\gamma: \mathbb{R} \rightarrow[0, \infty)$ such that

$$
\gamma(E)=\lim _{n \rightarrow \infty} \frac{1}{n} \log \|M(n, E, \theta)\| \text { for almost every } \theta,
$$

where $M(n, E, \theta)$ is the transfer matrix from 1 to $n$ for the operator $H_{\theta}$ at energy $E \in \mathbb{R}$, that is,

$$
M(n, E, \theta)=\left(\begin{array}{cc}
E-\lambda f\left(2^{n} \theta\right) & -1 \\
1 & 0
\end{array}\right) \times \cdots \times\left(\begin{array}{cc}
E-\lambda f(2 \theta) & -1 \\
1 & 0
\end{array}\right) .
$$

It is expected that $\gamma(E)>0$ for every $E$ and that $H_{\theta}$ has pure point spectrum with exponentially decaying eigenfunctions for almost every $\theta$. The reason for this is that the potentials are strongly mixing and similar to random potentials (generated by i.i.d. random variables) for which these results were established in the 1980's. There is a huge literature on this subject; we refer the reader to [4, 11] for detailed references.

However, very few results are known for the operators $H_{\theta}$ and these are limited to the case of small coupling: Chulaevsky and Spencer, [5, proved an asymptotic formula for $\gamma(E)$, in the limit $\lambda \rightarrow 0$. This implied positivity of $\gamma(E)$ for all $0<|E|<2$ and $\lambda$ small, which in turn was used by Bourgain and Schlag, $\underline{3}$, to prove that for $\lambda<\lambda_{0}$ and almost every $\theta, H_{\theta}$ has pure point spectrum in $0<\delta \leq|E| \leq 2-\delta$ with exponentially decaying eigenfunctions. This is usually referred to as Anderson localization.

Date: December 31, 2014.

D. D. was supported in part by NSF grant DMS-0227289. 
The results just described require a regularity assumption on $f$ (e.g., Hölder continuity). Beyond small coupling, no spectral results have been established. In particular, it was not known, for any $\lambda>0$, whether there can be any absolutely continuous spectrum (for a.e. $\theta$ ) 1 In this note, we show that there is none.

Theorem 1. Suppose that $\lambda>0$ and $f$ is measurable, bounded, and non-constant. Then, the Lyapunov exponent $\gamma(E)$ is positive for almost every $E \in \mathbb{R}$ and the absolutely continuous spectrum of $H_{\theta}$ is empty for almost every $\theta \in \mathbb{T}$.

Remark. Note that if the binary expansion of $\theta$ is periodic, the absolutely continuous spectrum of $H_{\theta}$ is not empty so that the second statement cannot be improved.

As we mentioned above, it is expected that $\gamma(E)$ is strictly positive for every energy $E$. Once this can be shown, the next natural step will then be to prove Anderson localization, using, for example, methods from [3. Since such a result is currently out of reach, we note that, by general principles, the almost everywhere positivity of $\gamma(E)$ yields the following consequence in terms of a localization result.

Given $\alpha \in[0, \pi)$, let $H_{\theta, \alpha}$ denote the operator which acts on $\ell^{2}\left(\mathbb{Z}_{+}\right)$as in (1), but with $\phi(0)$ given by $\cos (\alpha) \phi(0)+\sin (\alpha) \phi(1)=0$. Thus, the original operator (with a Dirichlet boundary condition) corresponds to $\alpha=0$. By [11, Theorem 13.4], Theorem 1 implies the following:

Corollary 1. Suppose that $\lambda>0$ and $f$ is measurable, bounded, and non-constant. Then, for almost every $\alpha \in[0, \pi)$ and almost every $\theta \in \mathbb{T}$, the operator $H_{\theta, \alpha}$ has pure point spectrum, and all eigenfunctions decay exponentially at infinity.

It is interesting to note that there are two possible viewpoints and both have been used successfully. The work of Chulaevsky and Spencer showed that methods from the random case (specifically an approach developed by Figotin and Pastur [11) could be extended to establish the asymptotic formula and positivity for the Lyapunov exponent. On the other hand, Anderson localization was then proven by Bourgain and Schlag by adapting methods originally developed for models with very little randomness, namely, with underlying dynamics given by the shift [1, 6, 7] and the skew-shift on the torus 2 .

The main ingredient in the proof of Theorem 1 will be a result of Kotani, 9] (see 12 for an adaptation to the discrete case), which shows that for whole-line operators with non-deterministic potentials, the Lyapunov exponent is almost everywhere positive and, consequently, the absolutely continuous spectrum is almost surely empty. A potential is non-deterministic if it is not determined uniquely by its restriction to a half-line. Thus, the natural strategy will be to define a nondeterministic family of whole-line operators whose restrictions to the right half-line yield the family $\left\{H_{\theta}\right\}$. Note that the Lyapunov exponents for the two families are the same and that the half-line operators cannot have absolutely continuous spectrum if the whole-line operators do not have any.

Proof of Theorem 1. The first step is to conjugate the doubling map $T$ to a symbolic shift via the binary expansion. Let $\Omega_{+}=\{0,1\}^{\mathbb{Z}_{+}}$and define $D: \Omega_{+} \rightarrow \mathbb{T}$ by

\footnotetext{
${ }^{1}$ It should be possible to exclude absolutely continuous spectrum using Kotani's support theorem [10]; see [5, 13] for remarks indicating this possibility and [8] for related applications of the support theorem. However, this would not give absence of absolutely continuous spectrum in full generality, that is, this approach would only work for some $f$ 's and some values of $\lambda$.
} 
$D(\omega)=\sum_{n=1}^{\infty} \omega_{n} 2^{-n}$. The shift transformation, $S: \Omega_{+} \rightarrow \Omega_{+}$, is given by $(S \omega)_{n}=$ $\omega_{n+1}$. Clearly, $D \circ S=T \circ D$.

Next we introduce a family of whole-line operators as follows. Let $\Omega=\{0,1\}^{\mathbb{Z}}$ and define, for $\omega \in \Omega$, the operator

$$
\left[H_{\omega} \phi\right](n)=\phi(n+1)+\phi(n-1)+V_{\omega}(n) \phi(n)
$$

in $\ell^{2}(\mathbb{Z})$, where

$$
V_{\omega}(n)=f\left[D\left(\left\{\omega_{n}, \omega_{n+1}, \omega_{n+2}, \ldots\right\}\right)\right] .
$$

The family $\left\{H_{\omega}\right\}_{\omega \in \Omega}$ is non-deterministic since $V_{\omega}$ restricted to $\mathbb{Z}_{+}$only depends on $\left\{\omega_{n}\right\}_{n \geq 1}$ and hence, by non-constancy of $f$, we cannot determine the values of $V_{\omega}(n)$ for $n \leq 0$ uniquely from the knowledge of $V_{\omega}(n)$ for $n \geq 1$. It follows from 9, 12 that the Lyapunov exponent for $\left\{H_{\omega}\right\}_{\omega \in \Omega}$ is almost everywhere positive and $\sigma_{\mathrm{ac}}\left(H_{\omega}\right)$ is empty for almost every $\omega \in \Omega$ with respect to the $\left(\frac{1}{2}, \frac{1}{2}\right)$-Bernoulli measure on $\Omega$.

Finally, let us consider the restrictions of $H_{\omega}$ to $\ell^{2}\left(\mathbb{Z}_{+}\right)$, that is, let $H_{\omega}^{+}=$ $E^{*} H_{\omega} E$, where $E: \ell^{2}\left(\mathbb{Z}_{+}\right) \rightarrow \ell^{2}(\mathbb{Z})$ is the natural embedding. Observe that $H_{\omega}^{+}=$ $H_{\theta}$, where $\theta=D\left(\left\{\omega_{1}, \omega_{2}, \omega_{2}, \ldots\right\}\right)$. This immediately implies the statement on the positivity of the Lyapunov exponent for the family $\left\{H_{\theta}\right\}_{\theta \in \mathbb{T}}$. As finite-rank perturbations preserve absolutely continuous spectrum, $\sigma_{\mathrm{ac}}\left(H_{\omega}^{+}\right) \subseteq \sigma_{\mathrm{ac}}\left(H_{\omega}\right)$ for every $\omega \in \Omega$. This proves that $\sigma_{\mathrm{ac}}\left(H_{\omega}^{+}\right)=\emptyset$ for almost every $\omega \in \Omega$.

Remark. The proof relies on only two properties of the doubling map: it is not one-to-one and it can be extended to a dynamical system over $\mathbb{Z}$. Consequently, Theorem 1 extends to other models with these properties. For example, $\theta \mapsto m \theta$ mod 1 for any integer $m \geq 2$.

Acknowledgments. We thank Svetlana Jitomirskaya and Barry Simon for useful conversations.

\section{REFERENCES}

[1] J. Bourgain and M. Goldstein, On nonperturbative localization with quasi-periodic potential, Ann. of Math. 152 (2000), 835-879

[2] J. Bourgain, M. Goldstein, and W. Schlag, Anderson localization for Schrödinger operators on $\mathbb{Z}$ with potentials given by the skew-shift, Commun. Math. Phys. 220 (2001), 583-621

[3] J. Bourgain and W. Schlag, Anderson localization for Schrödinger operators on $\mathbb{Z}$ with strongly mixing potentials, Commun. Math. Phys. 215 (2000), 143-175

[4] R. Carmona and J. Lacroix, Spectral Theory of Random Schrödinger Operators, Birkhäuser, Boston (1990)

[5] V. Chulaevsky and T. Spencer, Positive Lyapunov exponents for a class of deterministic potentials, Commun. Math. Phys. 168 (1995), 455-466

[6] M. Goldstein and W. Schlag, Hölder continuity of the integrated density of states for quasiperiodic Schrödinger equations and averages of shifts of subharmonic functions, Ann. of Math. 154 (2001), 155-203

[7] S. Jitomirskaya, Metal-insulator transition for the almost Mathieu operator, Ann. of Math. 150 (1999), 1159-1175

[8] W. Kirsch, S. Kotani, and B. Simon, Absence of absolutely continuous spectrum for some one-dimensional random but deterministic Schrödinger operators, Ann. Inst. H. Poincaré Phys. Théor. 42 (1985), 383-406

[9] S. Kotani, Ljapunov indices determine absolutely continuous spectra of stationary random one-dimensional Schrödinger operators, Stochastic analysis (Katata/Kyoto, 1982), pp. 225247, North-Holland Math. Library, 32, North-Holland, Amsterdam (1984)

[10] S. Kotani, Support theorems for random Schrödinger operators, Comm. Math. Phys. 97 (1985), 443-452 
[11] L. Pastur and A. Figotin, Spectra of Random and Almost-Periodic Operators, Grundlehren der Mathematischen Wissenschaften 297, Springer-Verlag, Berlin (1992)

[12] B. Simon, Kotani theory for one-dimensional stochastic Jacobi matrices, Commun. Math. Phys. 89 (1983), 227-234

[13] T. Spencer, Ergodic Schrödinger operators, in Analysis, et cetera, pp. 623-637, Academic Press, Boston (1990)

Mathematics 253-37, California Institute of Technology, Pasadena, CA 91125, USA

E-mail address: damanik@its.caltech.edu

Department of Mathematics, University of California, Los Angeles, CA 90055, USA

E-mail address: killip@math.ucla.edu 